\title{
Lower nutritional status and higher food insufficiency in frail older US adults
}

\author{
Ellen Smit $^{1 *}$, Kerrie M. Winters-Stone ${ }^{2}$, Paul D. Loprinzi ${ }^{3}$, Alice M. Tang ${ }^{4}$ and Carlos J. Crespo ${ }^{5}$ \\ ${ }^{1}$ School of Biological and Population Health Sciences, Oregon State University, Waldo 316, Corvallis, OR 97331, USA \\ ${ }^{2}$ Oregon Health E Science University, School of Nursing Portland Campus, 3455 SW US Veterans Road, SN-ORD, Portland, \\ OR 97239, USA \\ ${ }^{3}$ Department of Exercise Science, Donna \& Allan Lansing School of Nursing and Health Sciences, Bellarmine University, \\ 2001 Newburg Road, Louisville, KY 40205, USA \\ ${ }^{4}$ Department of Public Health and Community Medicine, Tufts School of Medicine, 136 Harrison Avenue, Jaharis 265, \\ Boston, MA O2111, USA \\ ${ }^{5}$ School of Community Health, Portland State University, Urban Center, Suite 450, 506 SW Mill Street, Portland, OR 97201 , \\ USA
}

(Submitted 23 January 2012 - Final revision received 26 July 2012 - Accepted 3 September 2012 - First published online 1 November 2012)

\begin{abstract}
Frailty is a state of decreased physical functioning and a significant complication of ageing. We examined frailty, energy and macronutrient intake, biomarkers of nutritional status and food insufficiency in US older adult (age $\geq 60$ years) participants of the Third National Health and Nutrition Examination Survey ( $n$ 4731). Frailty was defined as meeting $\geq 2$ and pre-frailty as meeting one of the following four-item criteria: (1) slow walking; (2) muscular weakness; (3) exhaustion and (4) low physical activity. Intake was assessed by $24 \mathrm{~h}$ dietary recall. Food insufficiency was self-reported as 'sometimes' or 'often' not having enough food to eat. Analyses were adjusted for sex, race, age, smoking, education, income, BMI, other co-morbid conditions and complex survey design. Prevalence of frailty was highest among people who were obese $(20 \cdot 8 \%)$, followed by overweight $(18 \cdot 4 \%)$, normal weight $(16 \cdot 1 \%)$ and lowest among people who were underweight (13.8\%). Independent of BMI, daily energy intake was lowest in people who were frail, followed by pre-frail and highest in people who were not frail (6648 (SE 130), 6966 (SE 79) and 7280 (SE 84) kJ, respectively, $P<0 \cdot 01$ ). Energy-adjusted macronutrient intakes were similar in people with and without frailty. Frail (adjusted OR (AOR) 4.7; 95\% CI 1.7, 12.7) and pre-frail (AOR 2.1; 95\% CI $0 \cdot 8$, 5.8) people were more likely to report being food insufficient than not frail people. Serum albumin, carotenoids and Se levels were lower in frail adults than not frail adults. Research is needed on targeted interventions to improve nutritional status and food insufficiency among frail older adults, while not necessarily increasing BMI.
\end{abstract}

Key words: Frailty: Nutritional status: Food insufficiency

Geriatric syndromes are a loosely defined group of conditions highly prevalent in older adults but not considered as discrete diseases; these include frailty, among others ${ }^{(1)}$. Frailty is a state of decreased physical functioning and a significant complication of ageing that increases the risk for incident falls, fractures, disability, co-morbidity, health care expenditure and premature mortality ${ }^{(2-4)}$. In 2010 , the proportion of US adults aged 65 years and older was $13 \%$; however, by 2030 it is projected that about $20 \%$ of the population will be older than 65 years of age, a 54\% increase. This shift in age demographics will have a substantial impact on the prevalence of frailty and highlights the need for identifying clinicaland population-based strategies to decrease the prevalence and consequences of frailty ${ }^{(5,6)}$.
Several studies have shown a potential association between nutrition and frailty. Specifically, low energy and protein intake and low serum nutrients have shown to be positively associated with frailty ${ }^{(7,8)}$. Studies have either been relatively small or with women only. Food insufficiency occurs when persons sometimes or often do not have enough food to eat. Food-insufficient older adults have been shown to have poorer dietary intake, nutritional status and health status than food-sufficient older adults ${ }^{(9)}$. Although little is known about food insufficiency as it relates to frailty, conceivably if food insufficiency is associated with poorer nutritional status, it may also be associated with physical functioning ${ }^{(10)}$ and frailty. Given the potential importance of nutrition on frailty and the amenability to intervention, we examine the

Abbreviations: InCHIANTI, Invecchiare in Chianti; NHANES III, Third National Health and Nutrition Examination Survey. 
association among nutritional status, food insufficiency and prevalent frailty in a representative sample of the US older adult population.

\section{Methods}

\section{Study participants}

The study population consists of adults aged 60 years and older, who took part in the Third National Health and Nutrition Examination Survey (NHANES III). Briefly, the NHANES III is a nationally representative sample of the population that used a stratified random sample of the civilian non-institutionalised population, drawn from fifty states in the USA and the District of Columbia during 1988-94 ${ }^{(11)}$. The analytic sample for the present study consists of older adults $(60+$ years) with complete data on each frailty criteria ( $n$ 4731). This particular survey period was chosen for the availability of frailty measures, which are not available in more recent survey years. The NHANES III was conducted according to the guidelines laid down in the Declaration of Helsinki, and all procedures involving human subjects were approved by the National Center for Health Statistics ethics committee. Written informed consent was obtained from all survey participants. The secondary data analysis for the present study was deemed exempt by the Oregon State University Institutional Review Board.

\section{Frailty}

A clinical definition of the frailty phenotype has been developed by Fried et al. ${ }^{(3)}$ for the general ageing population and is widely used and validated by others ${ }^{(4,12-14)}$. The definition is based on meeting three of the following five criteria: unintentional weight loss, slowness, muscle weakness, exhaustion and low physical activity. Given that our goal was to examine the association between energy intake and frailty, and because information on unintentional weight loss is not available in NHANES III, we excluded weight loss in our definition of frailty. This approach is similar to and modelled after the definition used by Bartali et $a{ }^{\left({ }^{(8)}\right.}$ in the Invecchiare in Chianti (InCHIANTI) study. InCHIANTI (Ageing in the Chianti area) is a community-based study of risk factors for disability performed in 1299 participants aged 65 years or older in Italy. The definition of frailty in the InCHIANTI study follows four of the five domains of frailty by Fried et $a l{ }^{(3)}$. Similarly, we defined frailty based on the four domains by Fried et al. ${ }^{(3)}$. These are: (1) slow walking, (2) muscular weakness, (3) exhaustion and (4) low physical activity. Participants were classified as frail if they met two or more of these four criteria. Participants who met one of the four criteria were classified as pre-frail and participants meeting none of the criteria were classified as not frail.

The specific measurement tools for each of the four frailty criteria were selected based on similar measurements or similar questions to those used by Fried et al. ${ }^{(3)}$ and the measurement tools that have been operationalised by other studies on frailty in NHANES III $^{(15-17)}$. A timed 8-foot walk test was performed twice and the best time (s) for the 8-foot walk was used for each participant. A participant was classified as a slow walker if their best time for the 8-foot walk was within the slowest quintile adjusted for sex. Participants were asked whether they had no difficulty, some difficulty, much difficulty or were unable to lift or carry something as heavy as 10 pounds (like a sack of potatoes or rice) when they were by themselves and without the use of aids. Participants who responded to this question as having some or much difficulty or unable to lift or carry that amount were classified as having muscular weakness. Participants were also asked whether they had no difficulty, some difficulty, much difficulty or were unable to walk from one room to another on the same level. Participants who responded to this question as having some or much difficulty or unable to walk from room to room were classified as having exhaustion. Participants who considered themselves as less active when compared with most men/women of the same age were classified as having low physical activity.

\section{Dietary intake and food insufficiency}

A $24 \mathrm{~h}$ recall was collected during the visit to the mobile examination centre. Dietary intake may differ by weekday, especially on weekend days. To capture intake on all days of the week, the $24 \mathrm{~h}$ recalls were collected on every day of the week. The dietary interviewers used the Dietary Data Collection system, which is an automated standardised interactive dietary interview and coding system. The system was specifically developed for NHANES III by the University of Minnesota Nutrition Coordinating Center ${ }^{(18)}$. Participants with $24 \mathrm{~h}$ recalls who were noted as incomplete or as unreliable during the interview were excluded ( $n$ 178). All the energy intake values from participants with reliable $24 \mathrm{~h}$ recalls were included in the final analysis, after preliminary analysis showed similar results with and without excluding outlying intakes (data not shown).

Food insufficiency was determined by asking participants if the food eaten by them and/or their families was enough food to eat, sometimes not enough food to eat or often not enough to eat. Respondents were considered to be food insufficient if they reported sometimes not enough food to eat or often not enough to eat. This has been found to be a reliable measure of food insufficiency ${ }^{(19)}$ and has been used by others ${ }^{(20,21)}$.

\section{Biochemical variables}

Blood concentrations were determined on a specimen obtained by venepuncture during the visit to the mobile examination centre. Details of the laboratory procedures can be found in the Laboratory Procedures Used in NHANES III ${ }^{(11)}$. Albumin was measured with the Boehringer Manneheim Diagnostics albumin system and the bromocresol purple binding agent. Folate was measured by using the Bio-Rad Laboratories 'Quantaphase Folate' radioassay kit. Serum levels of vitamins (vitamins $\mathrm{A}, \mathrm{B}_{12}$, C and $\mathrm{E}$, carotenoids) were measured by isocratic HPLC, with detection at three different wavelengths. Serum Se was measured using atomic absorption spectrophotometry. 


\section{Covariates}

We selected covariates based on known factors associated with frailty and/or nutritional status, including age, BMI, race-ethnicity, sex, smoking, education, income and presence of chronic diseases. Self-reported race and ethnicity were used to classify participants as non-Hispanic white, non-Hispanic black or Mexican-American (i.e. persons of Mexican origin living in the USA). Age was defined as the age in years at the time of the household interview. Education was based on the number of years the participant attended and completed school, and coded as less than high school, high school and more than high school. During the medical examination, height was measured using a stadiometer and weight was measured on a balance beam scale. Height and weight data were then used to calculate BMI (weight $(\mathrm{kg}) /$ height $\left.^{2}(\mathrm{~m})\right)$. BMI was categorised into underweight (BMI $<18.5 \mathrm{~kg} / \mathrm{m}^{2}$ ), normal weight (BMI $18.5-24.9 \mathrm{~kg} / \mathrm{m}^{2}$ ), overweight (BMI $25-29 \cdot 9 \mathrm{~kg} / \mathrm{m}^{2}$ ) and obese (BMI $\geq 30 \mathrm{~kg} / \mathrm{m}^{2}$ ) groups. Smoking history was assessed during the interview and classified into current, former or never smokers. For the present study, presence of current or history of chronic diseases related to physical function was based on affirmative responses to the following physician-diagnosed self-reported chronic conditions: lupus, osteoarthritis, rheumatoid arthritis, bronchitis, stroke, asthma, congestive heart failure, emphysema, heart attack, cancer and chronic low back pain.

\section{Analysis}

Total and adjusted means, variances and prevalences were calculated using multivariate linear and multinomial logistic regression models. Sample weights, provided by the National Center for Health Statistics, were used to correct for differential selection probabilities and to adjust for non-coverage and non-response. Logistic regression models with frailty as the outcome were used to obtain adjusted OR. Covariates that were significantly $(P<0.05)$ associated with frailty in the

Table 1. Characteristics of the Third National Health and Nutrition Examination Survey of adults aged 60 years and older by frailty (Weighted mean values or percentages with their standard errors)

\begin{tabular}{|c|c|c|c|c|c|c|c|}
\hline & \multicolumn{2}{|c|}{ Frail ( $n$ 1028) } & \multicolumn{2}{|c|}{ Pre-frail (n 1294) } & \multicolumn{2}{|c|}{ Not frail (n 2409) } & \multirow[b]{2}{*}{$P$} \\
\hline & Weighted mean & SE & Weighted mean & SE & Weighted mean & SE & \\
\hline Age (years) & $73 \cdot 1$ & 0.5 & $71 \cdot 8$ & 0.4 & $69 \cdot 4$ & 0.3 & $<0.001$ \\
\hline $\operatorname{Sex}(\%)$ & & & & & & & $<0.001$ \\
\hline Male & $35 \cdot 7$ & $2 \cdot 0$ & $36 \cdot 7$ & 1.9 & $46 \cdot 1$ & $1 \cdot 0$ & \\
\hline Female & $64 \cdot 3$ & $2 \cdot 0$ & $63 \cdot 3$ & 1.9 & 53.9 & 1.0 & \\
\hline Race-ethnicity (\%) & & & & & & & $<0.001$ \\
\hline White & 74.5 & $2 \cdot 6$ & $82 \cdot 7$ & 1.9 & $87 \cdot 8$ & $1 \cdot 2$ & \\
\hline Black & $14 \cdot 3$ & 1.8 & $10 \cdot 0$ & $1 \cdot 2$ & $6 \cdot 0$ & 0.7 & \\
\hline Mexican & $4 \cdot 1$ & 0.4 & $2 \cdot 6$ & 0.3 & $1 \cdot 7$ & 0.1 & \\
\hline Other & $7 \cdot 2$ & $2 \cdot 1$ & $4 \cdot 7$ & 1.4 & 4.5 & 0.9 & \\
\hline Education (\%) & & & & & & & $<0.001$ \\
\hline Less than high school & $59 \cdot 3$ & $3 \cdot 1$ & $44 \cdot 1$ & 2.9 & $35 \cdot 6$ & 1.9 & \\
\hline High school & $24 \cdot 7$ & $2 \cdot 0$ & $32 \cdot 1$ & 1.5 & $31 \cdot 7$ & 1.4 & \\
\hline More than high school & $16 \cdot 0$ & 2.5 & $23 \cdot 8$ & $2 \cdot 4$ & $32 \cdot 7$ & $1 \cdot 7$ & \\
\hline Income (\%) & & & & & & & $<0.001$ \\
\hline Less than $\$ 20000$ & $54 \cdot 2$ & 2.9 & $47 \cdot 1$ & $2 \cdot 8$ & $36 \cdot 3$ & $1 \cdot 7$ & \\
\hline$\$ 20000-34999$ & $17 \cdot 9$ & $2 \cdot 0$ & $21 \cdot 7$ & $2 \cdot 0$ & $28 \cdot 7$ & 1.3 & \\
\hline$\$ 35000$ or more & $27 \cdot 8$ & $2 \cdot 7$ & $31 \cdot 2$ & 2.4 & $35 \cdot 0$ & 1.9 & \\
\hline BMI status (\%) & & & & & & & $<0.001$ \\
\hline Underweight & $3 \cdot 3$ & 0.9 & $2 \cdot 3$ & 0.7 & $1 \cdot 7$ & 0.3 & \\
\hline Normal weight & $32 \cdot 3$ & 1.9 & $35 \cdot 8$ & $2 \cdot 1$ & $35 \cdot 6$ & $1 \cdot 7$ & \\
\hline Overweight & $32 \cdot 3$ & $2 \cdot 0$ & $36 \cdot 0$ & 1.9 & $41 \cdot 6$ & 1.4 & \\
\hline Obese & $32 \cdot 1$ & $2 \cdot 5$ & $25 \cdot 8$ & 1.5 & $21 \cdot 1$ & $1 \cdot 2$ & \\
\hline Employment (\%) & & & & & & & 0.02 \\
\hline Working & 1.5 & 0.8 & 0.8 & 0.4 & 2.9 & 0.6 & \\
\hline Retired & $67 \cdot 3$ & $2 \cdot 3$ & $68 \cdot 8$ & $2 \cdot 3$ & $71 \cdot 1$ & $1 \cdot 3$ & \\
\hline Keeping house & $26 \cdot 0$ & $2 \cdot 4$ & $27 \cdot 6$ & $2 \cdot 3$ & $23 \cdot 6$ & $1 \cdot 3$ & \\
\hline School or laid off & $5 \cdot 2$ & $1 \cdot 3$ & 2.7 & 0.8 & 2.4 & 0.4 & \\
\hline Smoking (\%) & & & & & & & $<0.01$ \\
\hline Current smoker & $19 \cdot 2$ & $2 \cdot 4$ & $16 \cdot 0$ & 1.6 & $14 \cdot 0$ & $1 \cdot 0$ & \\
\hline Previous smoker & 34.4 & $2 \cdot 6$ & $36 \cdot 7$ & $2 \cdot 2$ & $43 \cdot 1$ & 1.4 & \\
\hline Never smoked & $46 \cdot 4$ & $2 \cdot 2$ & $47 \cdot 2$ & $2 \cdot 2$ & $42 \cdot 9$ & 1.4 & \\
\hline Food insufficiency (\%) & & & & & & & $<0.001$ \\
\hline Secure & 94.9 & $1 \cdot 2$ & $98 \cdot 1$ & 0.5 & $99 \cdot 2$ & 0.2 & \\
\hline Insecure & $5 \cdot 1$ & $1 \cdot 2$ & 1.9 & 0.5 & 0.8 & 0.2 & \\
\hline Frailty criteria & & & & & & & NA \\
\hline Slow walking (\% yes) & $74 \cdot 1$ & $2 \cdot 3$ & $35 \cdot 7$ & 1.9 & 0.0 & & \\
\hline Weakness (\% yes) & $84 \cdot 6$ & 1.5 & $35 \cdot 3$ & $1 \cdot 7$ & 0.0 & & \\
\hline Exhaustion (\% yes) & $29 \cdot 9$ & $2 \cdot 2$ & $29 \cdot 2$ & 1.0 & 0.0 & & \\
\hline Low physical activity (\% yes) & 61.9 & $2 \cdot 4$ & $26 \cdot 1$ & 1.9 & 0.0 & & \\
\hline
\end{tabular}

NA, not applicable. 
univariate models were included in the multivariate models, namely age, BMI, race-ethnicity, sex, smoking, education, income and presence of chronic diseases. Additional models related to the macronutrients also adjusted for energy intake where appropriate. All analyses were completed using STATA (version 10.0, StataCorp LP).

\section{Results}

The prevalence of frailty in the US adults aged 60 years and older was $21.7 \%$, and the prevalence of pre-frailty was $27 \cdot 4 \%$. Characteristics of frail, pre-frail and not frail older adults are shown in Table 1. Among frail people, weakness was the most common criteria met, followed by slow walking, low physical activity and exhaustion (85, 74, 62 and 30\%, respectively). Frail people were older, less educated, at lower income levels, more likely to be female and current smokers and less likely to be white than adults who were not frail. Frail people were more likely to be underweight or obese than people who were not frail. In addition, frail people were more likely to report being food insufficient. In fact, people who were frail were 4.69 (95\% CI 1.73, 12.67) times more likely to report food insufficiency than people who were not frail, after adjusting for age, sex, race-ethnicity, smoking status, education, BMI and co-morbidity (data not shown). Pre-frail people were $2 \cdot 14$ (95\% CI $0 \cdot 79,5 \cdot 76)$ times more likely to report food insufficiency than people who were not frail. In general, pre-frail older adults had values for demographics, BMI and food insufficiency in between the values for frail and not frail adults. For example, the prevalence of obesity was $32 \%$ among frail people, $26 \%$ among pre-frail people and $21 \%$ among not frail people.

Energy intake was lowest in people who were frail, followed by pre-frail and highest in people who were not frail, independent of BMI (see Table 2). Crude dietary intake of grams of total fat, monounsaturated fat, carbohydrates and protein were lower in people who were frail (57.9 (SE 1.7), 21.7 (SE 0.6), 201 (SE 4.1) and 64.0 (SE 1.2), respectively) than people who were not frail (63.6 (SE 1.1), 24.4 (SE 0.4), 223 (SE 3.0) and 68.9 (se 0.7), respectively) (data not shown). However, after adjusting for differences in energy intake, intake of all macronutrients was similar among frail and not frail people (see Table 2). For example, protein intake, either as a percentage of total energy intake or as grams adjusted for energy intake, was similar for frail, pre-frail and not frail people. Plant and animal protein intakes were also similar. Biomarkers of nutritional status are shown in Table 3. Serum albumin, carotenoids and Se levels were significantly lower in people who were frail than people who were not frail.

To examine if one particular criterion for frailty accounts for most of the lower energy intake, we evaluated average energy intake between people who met or did not meet each of the four frailty criteria (see Table 4). Energy intake was lower in people who met each of the criteria than people who did not meet each of the respective criteria. For example, energy intake was lower in people who reported exhaustion than in people who reported no exhaustion. Similarly, underweight, obesity and food insufficiency were consistently higher in people who met each individual criterion than people who did not meet that particular criterion.

We examined frailty by BMI categories and found that the prevalence of frailty was highest among people who were obese $(20 \cdot 8 \%)$, followed by overweight (18.4\%), normal weight $(16 \cdot 1 \%)$ and lowest among people who were underweight $(13.8 \%)(P<0.01)$. We also compared energy intake levels for people who are frail and not frail by BMI categories. For each BMI category, energy intake was consistently lower in people who were frail than in people who were not frail

Table 2. Dietary intake of macronutrients in the Third National Health and Nutrition Examination Survey of adults aged 60 years and older by frailty $\dagger$

(Weighted mean values with their standard errors)

\begin{tabular}{|c|c|c|c|c|c|c|}
\hline & \multicolumn{2}{|c|}{ Frail (n 1028) } & \multicolumn{2}{|c|}{ Pre-frail ( $n$ 1294) } & \multicolumn{2}{|c|}{ Not frail ( $n$ 2409) } \\
\hline & Weighted mean & SE & Weighted mean & SE & Weighted mean & SE \\
\hline Energy $(\mathrm{kJ})^{\star}$ & 6648 & 128 & 6966 & 81 & 7286 & 84 \\
\hline Fat $(\mathrm{g}) \ddagger$ & $61 \cdot 8$ & 0.7 & $61 \cdot 3$ & 0.4 & $60 \cdot 8$ & 0.6 \\
\hline Percentage of energy & 31.8 & 0.4 & $31 \cdot 8$ & 0.2 & 31.9 & 0.4 \\
\hline SFA (g)‡ & $20 \cdot 9$ & 0.4 & $20 \cdot 4$ & 0.2 & $19 \cdot 9$ & 0.3 \\
\hline Percentage of energy & $10 \cdot 7$ & 0.2 & $10 \cdot 6$ & 0.1 & 10.5 & $0 \cdot 1$ \\
\hline MUFA $(g) \ddagger$ & $23 \cdot 3$ & 0.3 & $23 \cdot 3$ & 0.1 & $23 \cdot 3$ & 0.3 \\
\hline Percentage of energy & 11.9 & 0.2 & $12 \cdot 0$ & $0 \cdot 1$ & $12 \cdot 2$ & 0.2 \\
\hline PUFA (g)‡ & $12 \cdot 8$ & 0.4 & $12 \cdot 8$ & 0.2 & $12 \cdot 8$ & 0.2 \\
\hline Percentage of energy & $6 \cdot 6$ & 0.2 & $6 \cdot 6$ & 0.1 & $6 \cdot 7$ & $0 \cdot 1$ \\
\hline Carbohydrates (g)‡ & $211 \cdot 0$ & $2 \cdot 1$ & $213 \cdot 3$ & $1 \cdot 3$ & 215.5 & 1.6 \\
\hline Percentage of energy & $51 \cdot 7$ & 0.5 & 51.9 & 0.3 & $52 \cdot 0$ & 0.4 \\
\hline Protein (g)‡ & $67 \cdot 0$ & 0.8 & $66 \cdot 9$ & 0.5 & $66 \cdot 9$ & 0.6 \\
\hline Percentage of energy & $16 \cdot 5$ & 0.2 & $16 \cdot 4$ & 0.1 & $16 \cdot 3$ & 0.2 \\
\hline Animal protein $(\mathrm{g}) \ddagger$ & 64.5 & 0.8 & $64 \cdot 1$ & 0.5 & $63 \cdot 6$ & 0.5 \\
\hline Percentage of energy & $10 \cdot 8$ & 0.2 & $10 \cdot 7$ & 0.2 & $10 \cdot 6$ & 0.2 \\
\hline Plant protein $(\mathrm{g}) \ddagger$ & 35.5 & 0.8 & 35.9 & 0.5 & $36 \cdot 4$ & 0.5 \\
\hline Percentage of energy & $5 \cdot 2$ & $0 \cdot 1$ & $5 \cdot 3$ & 0.1 & 5.4 & 0.0 \\
\hline
\end{tabular}

${ }^{\star} P<0.001$.

†Adjusted for age, sex, race-ethnicity, smoking, education, income, BMI and co-morbidity.

$\ddagger$ Also adjusted for energy intake. 
Table 3. Serum biomarkers of nutritional status in the Third National Health and Nutrition Examination Survey of adults aged 60 years and older by frailty*

(Weighted mean values with their standard errors)

\begin{tabular}{|c|c|c|c|c|c|c|c|}
\hline & \multicolumn{2}{|l|}{ Frail } & \multicolumn{2}{|c|}{ Pre-frail } & \multicolumn{2}{|c|}{ Not frail } & \multirow[b]{2}{*}{$P$} \\
\hline & Weighted mean & SE & Weighted mean & SE & Weighted mean & SE & \\
\hline Albumin $(\mathrm{g} / \mathrm{l})(34-54 \mathrm{~g} / \mathrm{l}) \dagger$ & $39 \cdot 8$ & 0.0 & $40 \cdot 2$ & 0.0 & $40 \cdot 6$ & 0.0 & 0.002 \\
\hline Folate $(\mathrm{ng} / \mathrm{ml})(2 \cdot 7-17.0 \mathrm{ng} / \mathrm{ml}) \dagger$ & $9 \cdot 3$ & 0.6 & $9 \cdot 1$ & 0.3 & $8 \cdot 9$ & 0.3 & 0.84 \\
\hline $\operatorname{Se}(\mathrm{ng} / \mathrm{ml})(70-150 \mathrm{ng} / \mathrm{ml}) \dagger$ & $122 \cdot 6$ & 1.0 & $124 \cdot 2$ & 0.8 & $125 \cdot 8$ & 0.8 & 0.004 \\
\hline Total carotenoids $\ddagger(\mu \mathrm{g} / \mathrm{l})(480-2000 \mu \mathrm{g} / \mathrm{l}) \dagger$ & $751 \cdot 0$ & $18 \cdot 0$ & 788.0 & $11 \cdot 0$ & $825 \cdot 0$ & $15 \cdot 0$ & 0.01 \\
\hline Vitamin A $(\mu \mathrm{g} / \mathrm{l})(325-780 \mu \mathrm{g} / \mathrm{l}) \dagger$ & $637 \cdot 0$ & $11 \cdot 0$ & $637 \cdot 0$ & $7 \cdot 0$ & $636 \cdot 0$ & $6 \cdot 0$ & 0.43 \\
\hline Vitamin $B_{12}(\mathrm{pg} / \mathrm{ml})(200-900 \mathrm{pg} / \mathrm{ml}) \dagger$ & 471.1 & $19 \cdot 2$ & 487.5 & 11.7 & 503.9 & $20 \cdot 9$ & 0.57 \\
\hline Vitamin C (mg/l) $(2-20 \mathrm{mg} / \mathrm{l}) \dagger$ & $7 \cdot 7$ & 0.0 & $8 \cdot 1$ & 0.0 & 8.5 & 0.0 & 0.07 \\
\hline Vitamin E $(\mu \mathrm{g} / \mathrm{l})(5500-17000 \mu \mathrm{g} / \mathrm{l}) \dagger$ & $13521 \cdot 0$ & $311 \cdot 0$ & $13832 \cdot 0$ & $196 \cdot 0$ & $14142 \cdot 0$ & $249 \cdot 0$ & 0.07 \\
\hline
\end{tabular}

*Adjusted for age, sex, race-ethnicity, smoking, education, income, BMI and co-morbidity.

$\dagger$ Normal reference range.

$\ddagger$ Sum of $\beta$-carotene, $\beta$-cryptoxanthin, lutein/zeaxanthin and lycopene.

$(P<0 \cdot 01)$. The difference in energy intake between people who were frail and not frail was highest among people who were obese $(1367 \mathrm{~kJ})$, followed by overweight $(1294 \mathrm{~kJ})$, normal weight $(1220 \mathrm{~kJ})$ and lowest among people who were underweight $(1148 \mathrm{~kJ})$. Energy intakes by BMI category among pre-frail older adults were in between the values for frail and not frail adults (data not shown).

\section{Discussion}

We examined dietary intake and markers of nutritional status in relation to frailty in a population of US adults aged 60 years and older. Energy intake was lowest in frail, intermediate in pre-frail and highest in people who were not frail, independent of BMI. The present findings are in agreement with Bartali et al. ${ }^{(8)}$, who, using a similar definition of frailty as the present study, examined frailty in 802 persons aged 65 years and older in the InCHIANTI study. They reported energy intake to be lower in frail adults independent of BMI.

In contrast to other studies, we found that protein intake, either as energy-adjusted grams of protein or as a percentage of energy intake from protein, did not differ between people who were frail or not frail. The Women's Health Initiative observed that protein intake as a percentage of energy intake, but not absolute protein intake, was positively associated with the incidence of frailty in 24417 women aged 65-79 years $^{(7)}$. The InCHIANTI study reported that low protein intake, defined as the lowest quintile of intake in $\mathrm{g} / \mathrm{d}$, was positively associated with frailty ${ }^{(8)}$. Differences in the present findings may, in part, be due to differences in study design, study geographical location and analysis, where the Women's Health Initiative study examined the association of protein intake with the incidence of frailty in US women only and the InCHIANTI study reported prevalence of low protein (lowest quintile) intake by frailty status in Italy. NHANES III includes a single $24 \mathrm{~h}$ recall, which provides reasonable group means, but less reliably classifies people into low intakes of nutrients. Differences may also, in part, be due to differences in the frailty definition: the Women's Health Initiative study used the five domains of frailty including unintentional weight loss, while the present study and the InCHIANTI study used four domains excluding weight loss. We analysed the present results by including a fifth domain to reflect low BMI $\left(\mathrm{BMI}<18.5 \mathrm{~kg} / \mathrm{m}^{2}\right)$ in our definition of

Table 4. Energy intake, underweight and obesity, and food insufficiency for each of the frailty criteria in the Third National Health and Nutrition Examination Survey of adults aged 60 years and older*

(Weighted mean values with their standard errors and percentages)

\begin{tabular}{|c|c|c|c|c|c|}
\hline & \multicolumn{5}{|c|}{ Energy intake† } \\
\hline & Weighted mean & SE & Food insufficient† (\%) & Underweight (\%) & Obese (\%) \\
\hline \multicolumn{6}{|c|}{ Slow walking } \\
\hline Yes & 6770 & 121 & $37 \cdot 0$ & $2 \cdot 5$ & $29 \cdot 2$ \\
\hline No & 7201 & 84 & $19 \cdot 3$ & $2 \cdot 0$ & $22 \cdot 5$ \\
\hline \multicolumn{6}{|c|}{ Weakness } \\
\hline Yes & 6837 & 121 & $50 \cdot 2$ & 3.6 & $22 \cdot 5$ \\
\hline No & 7163 & 80 & $20 \cdot 4$ & 1.7 & $28 \cdot 4$ \\
\hline \multicolumn{6}{|c|}{ Exhaustion } \\
\hline Yes & 6489 & 293 & $16 \cdot 1$ & $4 \cdot 3$ & $33 \cdot 7$ \\
\hline No & 7125 & 75 & $4 \cdot 8$ & $2 \cdot 0$ & $23 \cdot 3$ \\
\hline \multicolumn{6}{|c|}{ Low physical activity } \\
\hline Yes & 6569 & 172 & $37 \cdot 7$ & 2.9 & $33 \cdot 3$ \\
\hline No & 7184 & 71 & $15 \cdot 1$ & $2 \cdot 0$ & $22 \cdot 1$ \\
\hline
\end{tabular}


frailty, as done by others ${ }^{(15,16)}$, and results did not change substantially (data not shown).

Along with lower energy intake, frail people were more likely to be food insufficient than not frail people. Food insufficiency reflects an inadequate amount of food due to lack of resources and, to our knowledge, has not been examined in relation to frailty by others. The lower energy intake and higher food insufficiency among people with frailty suggests that food sufficiency and energy intake may be important in the assessment and treatment of frailty. Further, the present findings of a higher prevalence of both underweight and obesity among people who are frail are also in agreement with other studies ${ }^{(22-24)}$. Hubbard et al. ${ }^{(22)}$ in the English Longitudinal Study on Ageing showed a U-shaped association between BMI and frailty. Similarly, Blaum et al. ${ }^{(23)}$ in the Women's Health and Aging Study showed a positive association between obesity and frailty. Thus, obesity does not preclude frailty, and the lower energy intake and higher food insufficiency in frail people occur across all categories of BMI.

The present findings of lower serum Se and carotenoids, and borderline lower levels of serum vitamins $\mathrm{C}$ and $\mathrm{E}$ are also in support of others ${ }^{(25-27)}$. Semba et al. ${ }^{(25)}$ using data from the Women's Health and Aging Study, found that women who were frail had lower serum Se and vitamin E. Similarly, lower serum albumin has been shown to be associated with greater loss of muscle mass ${ }^{(26)}$. The lower serum albumin among frail people in the present study may be an early indicator of impending muscle strength decline, as suggested by the results by Schalk et $a{ }^{(27)}$. Thus, the lower energy intake, lower serum albumin and lower serum nutrients indicate a lower nutritional status in people who are frail compared with people who are not frail. Energy balance may be present in persons who have both lower energy intake and lower physical activity. Low physical activity is one of the criteria of frailty, and energy intake was lower among people who were frail, suggesting potential energy balance in people who are frail. Regardless of the possible energy balance, frail people experienced lower serum nutrients and higher food insufficiency.

Strengths of the present study include dietary intake, serum nutrients and frailty measures on a representative sample of the civilian, non-institutionalised older US adult population and detailed data on important covariates. Limitations include the use of cross-sectional data, which limits our ability to assess cause-and-effect relationships. Nevertheless, these data are very useful in estimating frailty and describing nutritional status in the US population. Frailty measures were available for NHANES III (1988-94) and were not available for more recent survey years. Given the ageing population and the importance of physical function and nutrition in older adults, what we learn from NHANES III can be applicable today. The $24 \mathrm{~h}$ recall does not reflect usual or individual intake, yet it does provide reasonable group estimates of dietary intake ${ }^{(28)}$ for comparison of intake among frail, pre-frail and not frail people. The present estimates of food insufficiency are based on a single question and compared with more comprehensive measures may underestimate the prevalence of food insufficiency ${ }^{(29)}$. Pilot testing of the NHANES food insufficiency measure has shown it to be reliable ${ }^{(19)}$, and others have published results using these data ${ }^{(20,21)}$.

The definition of frailty was based on a modification of the Fried et $a l .{ }^{(3)}$ criteria to fit the available NHANES data, excluding unintentional weight loss. The criterion 'weakness' was based on interview data, as measured grip strength was not available in NHANES. Other criteria were based on interview questions in both Fried et al. ${ }^{(3)}$ and the present study, with questions varying slightly. The definition of frailty used in the present study is consistent with the definition of frailty used by others ${ }^{(15,16)}$ and has strong face validity. Moreover, the present study focused on energy intake and serum nutrients as a measure of nutritional status rather than weight loss. This approach allowed us to take a close look at the association between BMI and frailty.

\section{Conclusions}

In summary, we found that the prevalence of frailty increases with increasing BMI and that energy intake is consistently lower among frail than not frail older adults. Further, frail older adults are more likely to be food insufficient and have lower serum Se, carotenoids and albumin levels than older adults who are not frail. More research is needed on the energy balance of frailty, and on interventions that allow simultaneously improving nutritional status and food insufficiency among frail older adults, while not necessarily increasing BMI. In the meantime, the present results suggest that targeted interventions should focus on promoting availability and access to nutritious foods among older adults with frailty.

\section{Acknowledgements}

The work was partially supported by grants from the General Research Fund Award, Oregon State University (E. S.) and the National Institutes of Health (1 R25 GM086349-01: C. J. C.). E. S., K. M. W.-S. and C. J. C. designed the study; E. S. and P. D. L. analysed the data; K. M. W.-S., P. D. L. and A. M. T. interpreted the results and contributed to the writing of the paper; E. S. and C. J. C. wrote the paper; E. S. had responsibility for final content; all authors have approved the final manuscript. None of the authors claims a conflict of interest.

\section{References}

1. Inouye SK, Studenski S, Tinetti ME, et al. (2007) Geriatric syndromes: clinical, research, and policy implications of a core geriatric concept. J Am Geriatr Soc 55, 780-791.

2. Shardell M, Hicks GE, Miller RR, et al. (2009) Association of low vitamin D levels with the frailty syndrome in men and women. J Gerontol A Biol Sci Med Sci 64, 69-75.

3. Fried LP, Tangen CM, Walston J, et al. (2001) Frailty in older adults: evidence for a phenotype. J Gerontol A Biol Sci Med Sci 56, M146-M156.

4. Woods NF, LaCroix AZ, Gray SL, et al. (2005) Frailty: emergence and consequences in women aged 65 and older in the Women's Health Initiative Observational Study. $J \mathrm{Am}$ Geriatr Soc 53, 1321-1330. 
5. Crespo CJ, Smit E, Andersen RE, et al. (2000) Race/ethnicity, social class and their relation to physical inactivity during leisure time: results from the Third National Health and Nutrition Examination Survey, 1988-1994. Am J Prev Med 18, 46-53.

6. Vincent G \& Velkoff V (2010) The Next Four Decades: The Older Population in the United States 2010 to 2050. pp. 25-1128. Washington, DC: US Census Bureau, US Government Printing Office.

7. Beasley JM, LaCroix AZ, Neuhouser ML, et al. (2010) Protein intake and incident frailty in the Women's Health Initiative observational study. J Am Geriatr Soc 58, 1063-1071.

8. Bartali B, Frongillo EA, Bandinelli S, et al. (2006) Low nutrient intake is an essential component of frailty in older persons. J Gerontol A Biol Sci Med Sci 61, 589-593.

9. Lee JS \& Frongillo EA Jr (2001) Nutritional and health consequences are associated with food insecurity among U.S. elderly persons. J Nutr 131, 1503-1509.

10. Lee JS \& Frongillo EA Jr (2001) Factors associated with food insecurity among U.S. elderly persons: importance of functional impairments. J Gerontol B Psychol Sci Soc Sci 56, S94-S99.

11. The Third National Health and Examination Survey Reference Manuals and Reports. Hyattsville, MD: National Center for Health Statistics (CD-rom).

12. Graham JE, Snih SA, Berges IM, et al. (2009) Frailty and 10 -year mortality in community-living Mexican American older adults. Gerontology 55, 644-651.

13. Ahmed N, Mandel R \& Fain MJ (2007) Frailty: an emerging geriatric syndrome. Am J Med 120, 748-753.

14. Puts MT, Visser M, Twisk JW, et al. (2005) Endocrine and inflammatory markers as predictors of frailty. Clin Endocrinol (Oxf) 63, 403-411.

15. Wilhelm-Leen ER, Hall YN, Deboer IH, et al. (2010) Vitamin $\mathrm{D}$ deficiency and frailty in older Americans. J Intern Med 268, 171-180.

16. Smit E, Crespo CJ, Michael Y, et al. (2012) The effect of vitamin $\mathrm{D}$ and frailty on mortality among non-institutionalized US older adults. Eur J Clin Nutr 66, 1024-1028.

17. Wilhelm-Leen ER, Hall YN, K Tamura M, et al. (2009) Frailty and chronic kidney disease: the Third National Health and Nutrition Evaluation Survey. Am J Med 122, 664-671.e2.
18. Feskanich D, Sielaff BH, Chong K, et al. (1989) Computerized collection and analysis of dietary intake information. Comput Methods Programs Biomed 30, 47-57.

19. Briefel R \& Woteki C (1992) Development of food sufficiency questions for the Third National Health and Nutrition Examination Survey. J Nutr Educ 24, 24S-28S.

20. Dixon LB, Winkleby MA \& Radimer KL (2001) Dietary intakes and serum nutrients differ between adults from food-insufficient and food-sufficient families: Third National Health and Nutrition Examination Survey, 1988-1994. J Nutr 131, 1232-1246.

21. Alaimo K, Briefel RR, Frongillo EA, et al. (1998) Food insufficiency exists in the United States: results from the third National Health and Nutrition Examination Survey (NHANES III). Am J Public Health 88, 419-426.

22. Hubbard RE, Lang IA, Llewellyn DJ, et al. (2010) Frailty, body mass index, and abdominal obesity in older people. J Gerontol A Biol Sci Med Sci 65, 377-381.

23. Blaum CS, Xue QL, Michelon E, et al. (2005) The association between obesity and the frailty syndrome in older women: the Women's Health and Aging Studies. J Am Geriatr SoC 53, 927-934.

24. Bylow K, Hemmerich J, Mohile SG, et al. (2011) Obese frailty, physical performance deficits, and falls in older men with biochemical recurrence of prostate cancer on androgen deprivation therapy: a case-control study. Urology 77, 934-940.

25. Semba RD, Bartali B, Zhou J, et al. (2006) Low serum micronutrient concentrations predict frailty among older women living in the community. J Gerontol A Biol Sci Med Sci 61 , 594-599.

26. Visser M, Kritchevsky SB, Newman AB, et al. (2005) Lower serum albumin concentration and change in muscle mass: the Health, Aging and Body Composition Study. Am J Clin Nutr 82, 531-537.

27. Schalk BW, Deeg DJ, Penninx BW, et al. (2005) Serum albumin and muscle strength: a longitudinal study in older men and women. $J$ Am Geriatr Soc 53, 1331-1338.

28. Karvetti RL \& Knuts LR (1985) Validity of the 24-hour dietary recall. J Am Diet Assoc 85, 1437-1442.

29. Frongillo EA Jr, Rauschenbach BS, Olson CM, et al. (1997) Questionnaire-based measures are valid for the identification of rural households with hunger and food insecurity. J Nutr 127, 699-705. 